\title{
Early outcome of transurethral enucleation and resection of the prostate versus transurethral resection of the prostate
}

Sundaram Palaniappan ${ }^{1}$, MBBS, Tricia Li Chuen ${\underline{K u 0^{1}}}^{1}$, MBBS, FAMS, Christopher Wai Sam $\underline{\text { Cheng }}{ }^{1}$, FRCS, FAMS, Keong Tatt $\underline{\text { FOO }}^{1}$, FRCS, FAMS

\begin{abstract}
INTRODUCTION Recurrent prostate adenoma is a long-term complication following transurethral resection of the prostate (TURP). Transurethral enucleation and resection of the prostate (TUERP) is more appealing, since the nodular adenoma can be completely removed through endoscopy. TUERP is also hypothesised to result in a lower frequency of recurrent adenoma. This study aimed to compare the early outcomes of TUERP and TURP, and assess the feasibility and safety of TUERP.

METHODS We compared the outcome of 81 patients who underwent TUERP with that of 85 patients who underwent TURP. International prostate symptom score, quality of life score, prostate volume, degree of intravesical prostatic protrusion, maximum flow rate, post-void residual volume and prostate-specific antigen (PSA) level were obtained pre- and postoperatively. Complications (e.g. transfusion rate, incontinence, infection and urethral stricture) were analysed.

RESULTS Operative time was significantly longer in the TUERP group compared to the TURP group (85.3 minutes vs. 51.6 minutes). After TUERP, the maximum flow rate was significantly higher $(21.1 \mathrm{~mL} / \mathrm{s}$ vs. $17.1 \mathrm{~mL} / \mathrm{s})$ and PSA level was significantly lower $(1.2 \mathrm{ng} / \mathrm{mL}$ vs. $1.9 \mathrm{ng} / \mathrm{mL})$ than after TURP. The rates of infection, transfusion and urethral stricture were similar for both groups, but the TUERP group had a higher rate of temporary incontinence (13.6\% vs. $4.7 \%)$.

CONCLUSION The lower PSA level and better maximum flow rate achieved following TUERP suggest that prostate adenoma removal was more complete with TUERP. Long-term follow-up is required to establish whether TUERP results in fewer resections for recurrent adenoma.
\end{abstract}

Keywords: benign prostatic hyperplasia, transurethral enucleation and resection of prostate, transurethral resection of prostate

\section{INTRODUCTION}

Transurethral resection of the prostate (TURP) is the established gold standard surgical treatment for benign prostatic hyperplasia $(\mathrm{BPH})$. In the last few years, we have seen a growing number of minimally invasive procedures being developed to treat $\mathrm{BPH}_{\text {; }}$ these procedures, which are based on different technologies, aim to minimise morbidity while still maintaining the efficacy of TURP.

Rassweiler et al reported that the number of complications associated with TURP (e.g. clot retention, urinary tract infection and the need for blood transfusion) has declined over the years, and that transurethral resection syndrome is rare in clinical practice these days due to the advent of bipolar TURP, which allows for resection of the prostate with normal saline irrigation. ${ }^{(1)}$ However, the authors also highlighted that $3 \%-15 \%$ of patients required intervention five years after TURP for recurrence of $\mathrm{BPH} .{ }^{(1)}$ This is likely due to inadequate resection of the large prostate adenoma during initial surgery. In addition, TURP for large glands results in significantly more bleeding and clot retention as compared to open prostatectomy. It also requires a longer resection time, which leads to a higher risk of transurethral resection syndrome and substantial morbidity. ${ }^{(2,3)}$ For the aforementioned reasons, the guidelines published by the European Association of Urology recommend open prostatectomy over TURP in managing symptomatic large prostates (i.e. > 80-100 g). ${ }^{(4)}$ However, as open prostatectomy necessitates a lower abdominal incision, this may result in greater morbidity, a longer hospital stay and an extended recovery period.

This problem led to the development of a new technique, in which the practice of enucleation is adapted from open prostatectomy and incorporated into transurethral resection. This technique was first described in 1989 by Hiraoka and Akimoto, who used the tip of the resectoscope and a prostate-detaching blade to enucleate the prostate prior to its resection. ${ }^{(5)}$ This was then followed by a study by Liu et al in 2006, in which the authors performed a similar procedure using a bipolar resectoscope and no other additional equipment. ${ }^{(6)}$ The aim of the present study was to assess whether this new technique, termed transurethral enucleation and resection of the prostate (TUERP), was feasible, as well as to compare the early outcome of TUERP versus TURP.

\section{METHODS}

We retrospectively reviewed 81 consecutive patients who underwent TUERP (i.e. TUERP group) at the Department of Urology, Singapore General Hospital, Singapore, between March 2008 and January 2010. This cohort of patients was compared with 85 consecutive patients who underwent TURP (i.e. TURP group) in the same hospital between January 2006 and February 2008. 
Patients who had prostate cancer, neurogenic bladder and/or previous urethral or prostate surgery were excluded. The use of the $\mathrm{BPH}$ registry was approved by the Institutional Review Board of Singapore General Hospital.

All 166 patients had voiding symptoms that were attributed to $\mathrm{BPH}$. The diagnosis was confirmed after detailed history was taken and physical examinations, which included a digital rectal examination and transabdominal ultrasonography (TAUS), were performed. International prostate symptom score (IPSS) was used to grade the severity of the patient's voiding symptoms and assess the impact of the symptoms on the patient's quality of life (QOL). Uroflowmetry was done to measure the maximum flow rate (Qmax), and TAUS was performed to measure the prostate volume (PV), degree of intravesical prostatic protrusion (IPP) and post-void residual urine volume (PVR). Pre- and postoperative (six weeks after surgery) assessment of the PV was conducted by the same surgeon. PV was calculated using the ellipsoid formula (i.e. volume was calculated after the anteroposterior distance, transverse width and cephalocaudal height had been multiplied by 0.52). PV measurement was performed when the bladder was reasonably filled (i.e. 100-400 mL), as this has been shown to correlate well with transrectal PV measurement in a previous study. ${ }^{(7)}$

Indications for surgery included acute retention of urine, chronic retention, recurrent haematuria due to $\mathrm{BPH}$, urinary tract infection and bladder stones secondary to $\mathrm{BPH}$, failure of medical treatment, and significant obstruction. A patient was deemed to have significant obstruction if the PVR was persistently $>100 \mathrm{~mL}{ }^{(8)}$ usually with a reduced Qmax (i.e. $<10 \mathrm{~mL} / \mathrm{s}$ ). The following outcomes were measured in both groups of patients: time taken for resection of the prostate; weight of the resected prostate; number of days on bladder irrigation; number of days with indwelling catheter; and length of hospital stay. Postoperative IPSS, QOL score, Qmax, PVR, PV and serum prostate-specific antigen (PSA) level were measured during the patient's first follow-up visit to the clinic (six weeks after surgery). Patients were followed up for a variable length of time, depending on their symptoms, but the majority had at least six months of follow-up after discharge. Adverse events, such as the need for blood transfusion, sepsis following surgery, urethral stricture and urinary incontinence, were recorded. Further need for prostate surgery was also recorded if the patient re-presented to the surgeon with recurrent voiding symptoms suggestive of prostate adenoma regrowth.

Both TURP and TUERP were performed using the plasmakinetic bipolar system with normal saline irrigation, under spinal or general anaesthesia. All surgeries were performed or closely supervised by the same surgeon. Preprogrammed power settings for cutting $(180 \mathrm{~W})$ and coagulation $(80 \mathrm{~W})$ were used. Preliminary cystoscopy was done using a 20-F-sized sheath, to assess both prostate size and shape, and visualise landmarks (including the two ureteric orifices and the verumontanum). A 26-F-sized resectoscope was then introduced, and the TUERP procedure was performed as described in the following paragraph.

The distal margin of the prostate lobes was marked with a cutting loop. Starting with the median lobe, the mark between
Table I. Baseline characteristics of the patients who underwent TURP or TUERP.

\begin{tabular}{lccc}
\hline Variable & \multicolumn{2}{c}{ Mean (range) } & p-value \\
\cline { 2 - 3 } & $\begin{array}{c}\text { TURP group } \\
(\mathbf{n}=\mathbf{8 5})\end{array}$ & $\begin{array}{c}\text { TUERP group } \\
\mathbf{( n = 8 1 )}\end{array}$ & \\
\hline Age $(\mathrm{yr})$ & $68.3(50-94)$ & $70.3(48-92)$ & 0.28 \\
PV $(\mathrm{mL})$ & $53.7(13-152)$ & $53.0(11-158)$ & 0.87 \\
PSA level $(\mathrm{ng} / \mathrm{mL})$ & $6.7(0.3-47.6)$ & $10.7(1.0-64.6)$ & 0.06 \\
IPSS & $16.3(4-35)$ & $13.3(2-29)$ & 0.05 \\
QOL score & $3.6(1-6)$ & $3.1(0-6)$ & 0.05 \\
Qmax $(\mathrm{mL} / \mathrm{s})$ & $9.6(2.4-23.0)$ & $7.9(1.1-29.3)$ & 0.17 \\
PRV $(\mathrm{mL})$ & $91.3(0-300)$ & $108.5(0-300)$ & 0.18 \\
\hline
\end{tabular}

IPSS: international prostate symptom score; PRV: post-void residual urine volume; PSA: prostate-specific antigen; PV: prostate volume; Qmax: maximum flow rate; QOL: quality of life; TUERP: transurethral enucleation and resection of the prostate; TURP: transurethral resection of the prostate

the 5 o'clock and 7 o'clock positions was deepened, down to the surgical capsule. Next, the incision just proximal to the verumontanum was deepened so that the plane between the adenoma and false capsule (i.e. the compressed normal prostatic tissue) could be identified with a thick resectoscope loop. The tip of the resectoscope was then inserted to further develop this plane. The adenoma was gradually dissected away from the capsule in the avascular plane, toward the bladder neck, until the circular fibres of the bladder neck were seen. The perforating vessels were diathermied at the source and cut. Bleeding points noted during this mechanical dissection were easily controlled with coagulation. The median lobe, which was still attached to the bladder neck, was then resected. The plane between the lateral lobe and false capsule was developed in a similar manner and resected. No morcellator was used. The chips were then evacuated using a bladder evacuator. On completion, a three-way Foley catheter was inserted and continuous bladder irrigation was initiated. Bladder irrigation was kept overnight or until the effluent was clear. Once the irrigation was stopped, the catheter was removed after two days, or when the urine was clear or had only a light pink colouration.

The baseline characteristics and perioperative data of the two groups were compared using Student's t-test, while the incidence of postoperative adverse events was compared using chi-square test. A p-value $<0.05$ was considered to be statistically significant in all analyses.

\section{RESULTS}

The baseline characteristics of all the patients are shown in Table I. No significant differences were found between the patients in the TURP and TUERP groups in terms of age, PV, PSA level, IPSS, QOL score, Qmax and PVR. Table II shows the perioperative data of the patients. The duration of bladder irrigation was significantly higher for the patients who underwent TURP compared to those who underwent TUERP (1.17 days vs. 1.00 days, $p=0.03$ ); this is likely related to the better haemostasis achieved with the TUERP procedure. However, the duration of indwelling catheter and length of hospital stay were not lower in the TUERP group. Although the weights of 
Table II. Perioperative data of the patients who underwent TURP or TUERP.

\begin{tabular}{|c|c|c|c|}
\hline \multirow[t]{2}{*}{ Variable } & \multicolumn{2}{|c|}{ Mean (range) } & \multirow[t]{2}{*}{ p-value } \\
\hline & TURP group $(n=85)$ & TUERP group $(n=81)$ & \\
\hline Weight of resected prostate chips (g) & $32.2(2-145)$ & $29.7(3-73)$ & 0.12 \\
\hline Resection time (min) & $51.6(9-150)$ & $85.3(20-190)$ & $<0.01^{*}$ \\
\hline Duration of bladder irrigation (day) & $1.17(0-3)$ & $1.00(1)$ & $0.03^{*}$ \\
\hline Duration of indwelling catheter (day) & $2.07(1-7)$ & $2.26(1-8)$ & 0.27 \\
\hline Postoperative PV (mL) & $14.7(5-41)$ & $14.9(5-39)$ & 0.67 \\
\hline Postoperative PSA level (ng/mL) & $1.9(0.3-7.7)$ & $1.2(0.1-6.3)$ & $0.01^{*}$ \\
\hline IPSS & $7.1(1-29)$ & $6.9(0-25)$ & 0.80 \\
\hline QOL score & $1.8(0-6)$ & $1.5(0-6)$ & 0.48 \\
\hline $\mathrm{Qmax}(\mathrm{mL} / \mathrm{s})$ & $17.1(4.9-43.0)$ & $21.1(5.7-39.5)$ & $<0.01^{*}$ \\
\hline PRV $(m L)$ & $27.2(0-167)$ & $32.5(0-100)$ & 0.13 \\
\hline
\end{tabular}

*Statistically significant at $\mathrm{p}<0.05$. IPSS: international prostate symptom score; PRV: post-void residual urine volume; PSA: prostate specific antigen; PV: prostate volume; Qmax: maximum flow rate; QOL: quality of life; TUERP: transurethral enucleation and resection of the prostate; TURP: transurethral resection of the prostate

Table III. Complications observed among the patients during the follow-up period, according to the type of surgery.

\begin{tabular}{lccc}
\hline Variable & \multicolumn{2}{c}{ No. (\%) } & p-value \\
\cline { 2 - 3 } & TURP ( $\mathbf{n = 8 5 )}$ & TUERP ( $\mathbf{n = 8 1 )}$ & \\
\hline Blood transfusion & $2(2.4)$ & $3(3.7)$ & 0.68 \\
Urinary sepsis & $2(2.4)$ & $1(1.2)$ & 1.00 \\
Urethral stricture & $5(5.9)$ & $2(2.5)$ & 0.44 \\
Incontinence & $4(4.7)$ & $11(13.6)$ & 0.06 \\
\hline
\end{tabular}

TUERP: transurethral enucleation and resection of the prostate; TURP: transurethra resection of the prostate

prostate chips resected from the patients in the TUERP and TURP groups were similar (29.7 g vs. $32.2 \mathrm{~g}, \mathrm{p}=0.12)$, the resection time was found to be significantly longer for the TUERP group (85.3 minutes vs. 51.6 minutes, $\mathrm{p}<0.01$ ). The longer resection time may be due to the steep learning curve associated with any new surgical procedure. No statistical difference was noted between the two groups in terms of the patients' postoperative IPSS, QOL score, PVR and PV. The postoperative serum PSA level was significantly higher in the TURP group than in the TUERP group $(1.9 \mathrm{ng} / \mathrm{mL}$ vs. $1.2 \mathrm{ng} / \mathrm{mL}, \mathrm{p}=0.01)$ and improvement in Qmax was significantly better for the TUERP group $(21.1 \mathrm{~mL} / \mathrm{s}$ vs. $17.1 \mathrm{~mL} / \mathrm{s}, \mathrm{p}<0.01)$.

The median duration of follow-up for the patients in the TURP group was 8 (range $2-108$ ) months, while the corresponding duration for the patients in the TUERP group was 11 (range 2-85) months. The complications observed during the follow-up period are shown in Table III. No significant difference was found between the two groups in terms of the rates of transfusion, urethral stricture and urinary sepsis. Even though the number of patients with urinary incontinence was higher in the TUERP group than in the TURP group (11 vs. four), this difference was not statistically significant. The incontinence was commonly urge-related and transient in all cases, with all of the patients showing gradual improvement over six months. In addition, the urge incontinence could have been secondary to preoperative benign prostatic obstruction; storage symptoms arising from this cause have been known to improve gradually after surgery.

\section{DISCUSSION}

The evolution of TURP to include enucleation was deemed necessary, as TURP had a higher re-operation rate than open prostatectomy. Between $12 \%$ and $15.5 \%$ of patients who underwent TURP required a second operation, while only $1.8 \%-4.5 \%$ of patients who underwent open prostatectomy required a second one. ${ }^{(9)}$ This difference could be related to the inadequate excision of the prostate adenoma in TURP as compared to open prostatectomy. Transurethral enucleation of the prostate was first developed by Hiraoka and Akimoto; in their described method, a prostate-detaching blade was used to assist in the detachment of the adenoma from the surgical capsule. ${ }^{(5)}$ Neill et al later proposed the use of bipolar plasmakinetic energy as an alternative energy source to enucleate the prostate; this method is similar to holmium laser enucleation of the prostate (HoLEP), ${ }^{(10)}$ a procedure that is becoming established as the endoscopic alternative to open prostatectomy. After enucleation of the adenoma with the holmium laser, the cavity in the prostate is comparable to that achieved after open prostatectomy. According to a meta-analysis, HoLEP is the only minimally invasive procedure that is more efficacious than TURP; recent long-term follow-up data also proved that HoLEP is a durable procedure. ${ }^{11,12}$

In a similar manner to holmium energy in enucleation of the prostate adenoma, plasmakinetic energy could be used. The technique used in the present study has been described by Liu et al. In this technique, a plasmakinetic device is exclusively used to perform the procedure - from enucleation of the adenoma and coagulation of the bleeding vessels during dissection to resection of the adenoma tissue that is still partially attached to the bladder neck. ${ }^{(6)}$ Liu et al's review of 1,100 patients who underwent this procedure showed improvements in Qmax (to $24.3 \mathrm{~mL} / \mathrm{s}$ ), reduction in PVR and improvement in symptom scores over 4.3 years of follow-up. ${ }^{(6)}$ Recently, another study compared the results of TUERP with TURP and found that patients from both groups had smaller PV (10.5 g vs. $15.2 \mathrm{~g}$ ) after the procedures; it also found that the postoperative PSA level of patients who underwent TUERP was significantly lower than that of patients who underwent TURP (0.8 ng/mL vs. $2.8 \mathrm{ng} / \mathrm{mL})$;(13) however, 
the patients in the two groups were operated on at different institutions, by different surgeons.

In our cohort of patients who underwent surgical management of $\mathrm{BPH}$, there were no significant differences in $\mathrm{PV}$, symptom scores, PSA level, Qmax and PVR. Our results showed that symptomatic improvement (measured using IPSS and QOL score) following TUERP was equivalent to that following TURP. In addition, postoperative PV and PVR (measured during follow-up at the clinic) did not differ significantly between the two groups. This suggests that no significant obstruction was present after TUERP (subjectively, based on the symptom scores, and objectively, based on postoperative PV and PVR). Ultrasonographic assessments of prostate size, intravesical prostatic protrusion and $\mathrm{PVR}^{(14,15)}$ are considered to be a surrogate for the degree of bladder outlet obstruction. Postoperative PV is difficult to interpret, as it does not correspond well to the actual residual PV since there is a cavity after prostate resection. This may explain the lack of difference in postoperative PV. Although we did not observe any significant difference in the mean resected volume, this may have been due to the small number of patients in the present study. Moreover, the volume of resected prostate is not as important as the volume of adenoma left behind. In contrast, Qmax was found to be significantly higher in the TUERP group compared to the TURP group $(21.1 \mathrm{~mL} / \mathrm{s}$ vs. $17.1 \mathrm{~mL} / \mathrm{s}, \mathrm{p}<0.01)$. Uroflowmetry is a more objective way of assessing postoperative obstruction than PVR measurement; this is because PVR may vary significantly due to premicturition volume and the timing of the measurement. Hence, the greater Qmax seen among the patients who underwent TUERP suggests that TUERP was more effective in removing the obstructing adenoma than TURP.

The postoperative PSA level of the patients in the TUERP group was significantly lower than that of the patients in the TURP group (1.2 ng/mL vs. $1.9 \mathrm{ng} / \mathrm{mL}, \mathrm{p}=0.01)$. Furthermore, the reduction in mean PSA level for the TUERP group was $88.8 \%$, while it was only $71.6 \%$ for the TURP group. The raised PSA level seen in $\mathrm{BPH}$ is proposed to be due to hyperplasia of the transition zone. Hence, if there was complete resection of the adenoma, the PSA level should return to the normal range (i.e. below $4 \mathrm{ng} / \mathrm{mL}$ ). ${ }^{(16)}$ Thus, the greater reduction in mean PSA level in the TUERP group indicated that adenoma resection was more complete with TUERP than with TURP. ${ }^{(17)}$ The lower PSA level and higher peak urinary flow can be considered as surrogate markers of complete adenoma removal. However, the most important assessment is whether these patients eventually developed recurrence of the prostate adenoma. This data, however, was not available in the present study due to its short follow-up period. A longer follow-up period (i.e. $>5$ years) may be necessary to find out whether there is a difference in the recurrence rates of prostate adenoma between patients who underwent TUERP and those who underwent conventional TURP.

In the present study, the time taken for resection was notably longer in TUERP than in TURP. This is not surprising, as TUERP requires time for both enucleation and resection. Furthermore, the patients who underwent TUERP were part of our early experience in this procedure and, hence, there was a relatively steep learning curve associated with the acquisition of the technique for enucleation. The main hurdles include identification of the cleavage plane between the false capsule and adenoma, as well as performing blunt detachment of the adenoma in a retrograde manner (i.e. the crucial steps in TUERP). ${ }^{(18)}$ With experience, the resection time for TUERP should improve.

We did not observe any significant increase in the incidence of complications (e.g. blood transfusion, urinary sepsis and urethral stricture) among both groups of patients. However, there was a significantly higher percentage of patients who developed urinary incontinence following TUERP. This could be explained by the need for more extensive dissection around the external sphincter region during the identification of the cleavage plane in TUERP. Both the complete removal of the prostate adenoma and disuse of the external sphincter could have led to the weakening of the external sphincter, leading to incontinence. ${ }^{(19)}$ However, the incontinence was temporary and resolved in all the patients after six months. In terms of adenoma recurrence, both the TUERP and TURP groups had a similar number of patients who presented with recurrence, although the adenoma was not significant enough to warrant a repeat resection.

Based on our early experience with TUERP, the ideal or index patient for this procedure would be one whose preoperative assessment showed a prostate size of 40-80 g on ultrasonography. This is because the plane between the adenoma and false capsule is better defined in a large prostate than in a small one (i.e. $<40 \mathrm{~g}$ ). A small prostate may have a less well-defined plane, possibly due to chronic inflammation. Large prostates that weigh $>80 \mathrm{~g}$ can be challenging, as during enucleation, potential spaces are created, which may cause a beginner to be disorientated. To overcome this problem, the surgeon can resort to enucleation and resection at the same time. There is no real necessity to complete enucleation before resection of the adenoma.

In the present study, there were too few patients with a prostate size $>80 \mathrm{~g}$ in both groups to make a meaningful comparison. With bipolar resection using normal saline, transurethral resection syndrome is no longer a problem. Even though resection time may be prolonged for large prostates, they can still be operated on. The more immediate advantage of TUERP is that haemostasis can be better achieved, as the perforating vessels are diathermied at the source instead of repeatedly resected (as in conventional TURP).

There are limitations to the present study. First, we used a retrospective study design and the analysis was done using a relatively small sample size. Additionally, as the period of follow-up was short, we were unable to comment on the longterm outcomes of TUERP, particularly its effects on prostate adenoma recurrence. Long-term follow-up data was not available for all the patients, as most of the patients who underwent transurethral surgery were discharged from follow-up about six months after surgery. Nevertheless, the present study provides evidence that TUERP is a safe and efficacious treatment option for larger prostates. Undoubtedly, a randomised prospective trial with a longer follow-up period would be useful in determining 
whether patients who undergo TUERP have fewer re-operations than patients who undergo TURP.

In the present study, we showed that TUERP has equivalent early outcomes as TURP in terms of symptom improvement and PVR. Patients who underwent TUERP achieved better peak urinary flow and lower postoperative PSA level, suggesting that TUERP results in more complete adenoma removal than TURP. While any attempt to remove a prostate adenoma via endoscopic means such as open simple prostatectomy is fundamentally possible, it remains to be seen whether the procedure results in less adenoma recurrence. Further studies with long-term follow-up are needed to ascertain this.

\section{REFERENCES}

1. Rassweiler J, Teber D, Kuntz R, Hofmann R. Complications of transurethral resection of the prostate (TURP)--incidence, management, and prevention. Eur Urol 2006; 50:969-79; discussion 980.

2. Persu C, Georgescu D, Arabagiu I, et al. TURP for BPH. How large is too large? J Med Life 2010; 3:376-80.

3. Simforoosh $\mathrm{N}$, Abdi $\mathrm{H}$, Kashi $\mathrm{AH}$, et al. Open prostatectomy versus transurethral resection of the prostate, where are we standing in the new era? A randomized controlled trial. Urol J 2010; 7:262-9.

4. Gravas S, Bach T, Bachmann A, et al. Guidelines on the management of non-neurogenic male lower urinary tract symptoms (LUTS), incl. benign prostatic obstruction (BPO). In: European Association of Urology [online]. Available at: http://uroweb.org/wp-content/uploads/EAU-GuidelinesNon-Neurogenic-Male-LUTS-Guidelines-2015-v2.pdf. Accessed February 1, 2016.

5. Hiraoka Y, Akimoto M. Transurethral enucleation of benign prostatic hyperplasia. J Urol 1989; 142:1247-50.

6. Liu C, Zheng S, Li H, Xu K. Transurethral enucleation and resection of prostate in patients with benign prostatic hyperplasia by plasma kinetics. J Urol 2010; 184:2440-5.

7. Yuen JS, Ngiap JT, Cheng CW, Foo KT. Effects of bladder volume on transabdominal ultrasound measurements of intravesical prostatic protrusion and volume. Int J Urol 2002; 9:225-9.
8. Wang D, Foo KT. Staging of benign prostate hyperplasia is helpful in patients with lower urinary tract symptoms suggestive of benign prostate hyperplasia. Ann Acad Med Singapore 2010; 39:798-802.

9. Roos NP, Wennberg JE, Malenka DJ, et al. Mortality and reoperation after open and transurethral resection of the prostate for benign prostatic hyperplasia. N Engl J Med 1989; 320:1120-4.

10. Neill MG, Gilling PJ, Kennett KM, et al. Randomized trial comparing holmium laser enucleation of prostate with plasmakinetic enucleation of prostate for treatment of benign prostatic hyperplasia. Urology 2006; 68:1020-4.

11. Tan AH, Gilling PJ, Kennett KM, et al. A randomized trial comparing holmium laser enucleation of the prostate with transurethral resection of the prostate for the treatment of bladder outlet obstruction secondary to benign prostatic hyperplasia in large glands (40 to 200 grams). J Urol 2003; 170:1270-4.

12. Ahyai SA, Gilling P, Kaplan SA, et al. Meta-analysis of functional outcomes and complications following transurethral procedures for lower urinary tract symptoms resulting from benign prostatic enlargement. Eur Urol 2010; 58:384-97.

13. Zhang KY, Xing JC, Chen BS, et al. Bipolar plasmakinetic transurethral resection of the prostate vs. transurethral enucleation and resection of the prostate: pre-and postoperative comparisons of parameters used in assessing benign prostatic enlargement. Singapore Med J 2011; 52:747-51.

14. Chia SJ, Heng CT, Chan SP, Foo KT. Correlation of intravesical prostatic protrusion with bladder outlet obstruction. BJU Int 2009; 91:371-4.

15. Foo KT. Decision making in the management of benign prostatic enlargement and the role of transabdominal ultrasound. Int J Urol 2010; 17:974-9.

16. Furuya $\mathrm{Y}$, Akakura $\mathrm{K}$, Tobe $\mathrm{T}$, et al. Changes in serum prostate-specific antigen following prostatectomy in patients with benign prostate hyperplasia. Int J Urol 2000; 7:447-51.

17. Tinmouth WW, Habib E, Kim SC, et al. Change in serum prostate specific antigen concentration after holmium laser enucleation of the prostate: a marker for completeness of adenoma resection? J Endourol 2005; 19:550-4.

18. Xiong W, Sun $M$, Ran Q, et al. Learning curve for bipolar transurethral enucleation and resection of the prostate in saline for symptomatic benign prostatic hyperplasia: experience in the first 100 consecutive patients. Urol Int 2013; 90:68-74.

19. Shah HN, Mahajan AP, Hegde SS, Bansal MB. Peri-operative complications of holmium laser enucleation of the prostate: experience in the first 280 patients, and a review of literature. BJU Int 2007; 100:94-101. 